\title{
Miller Fisher Variant of Guillain-Barré Syndrome Triggered by Ventilator-Associated Pneumonia
}

\author{
Danah Aljaafari' \\ Salam Almustafa ${ }^{2}$ \\ Abdulrahman Saleh $\mathrm{Ali}^{3}$ \\ Hosam Aldalbahi ${ }^{3}$ \\ Norah Ibrahim Albahli ${ }^{3}$ \\ Feras AISulaiman (D) \\ Anas Al dehailan' \\ Majed Alabdali' \\ 'Department of Neurology, College of \\ Medicine, Imam Abdulrahman Bin Faisal \\ University, Dammam, Saudi Arabia; \\ ${ }^{2}$ College of Medicine, Imam Abdulrahman \\ Bin Faisal University, Dammam, Saudi \\ Arabia; ${ }^{3}$ Department of Neurology, King \\ Fahad Medical City, Riyadh, Saudi Arabia
}

Correspondence: Danah Aljaafari Department of Neurology, College of Medicine Imam Abdulrahaman Bin Faisal University, 2835 King Faisal Road, Dammam, 34212, Kingdom of Saudi Arabia Tel +966503864084
Background: Miller Fisher syndrome (MFS), a triad of ophthalmoplegia, areflexia and ataxia, is one of the regional variants of Guillain-Barré syndrome (GBS) that might account for a quarter of all cases of GBS, especially in Asian countries. There is history of an antecedent upper respiratory tract infection in up to two thirds of MFS cases. However, association of MFS in adults and pneumonia is rarely reported and in those cases causative pathogen was Mycoplasma pneumoniae. To our knowledge, association of MFS and ventilator-associated pneumonia has never been reported. So, we hereby report the first case of MFS which followed ventilator-associated pneumonia (VAP).

Case Report: We report case of a 22-year-old male who was known to have temporal lobe epilepsy and mental retardation. He presented with status epilepticus. He was sedated and put on mechanical ventilation. Two days later, he developed a fever associated with increased tracheobronchial secretions and new infiltrates on chest X-ray. Diagnosis of VAP was made. Upon improvement, he was extubated and shifted out of ICU. Ten days after the onset of fever, he developed gradual onset bulbar weakness and ataxia. On examination, he had generalized areflexia and ataxia. CSF analysis showed cytoalbuminic dissociation. Antibodies against ganglioside complex were elevated. Diagnosis of sero-negative MFS was made, and intravenous immunoglobulin (IVIG) was started. He improved remarkably within two days.

Conclusion: MFS is immune-mediated entity which is usually triggered by upper respiratory tract infection but in rare cases it can be consequence of pneumonia including VAP. Further research is needed to establish link between MFS and VAP.

Keywords: Guillain-Barré syndrome, Miller Fisher syndrome, ventilator-associated pneumonia, Pseudomonas aeruginosa

\section{Introduction}

Miller Fisher syndrome (MFS), a clinical triad of ophthalmoplegia, areflexia and ataxia, is one of the regional variants of Guillain-Barré syndrome (GBS). It may account for a quarter of all cases of GBS, especially in Asian countries. There is a history of an antecedent upper respiratory tract infection in up to two thirds of MFS cases. $^{1,2}$ Most common antecedent infections being reported in patients with MFS are Campylobacter jejuni followed by Haemophilus influenzae and Cytomegalovirus, in that order. ${ }^{3}$ However, the association of MFS in adults and pneumonia is rarely reported and in those cases the causative pathogen was Mycoplasma pneumonia., ${ }^{4,5}$ To our knowledge, association of MFS and ventilatorassociated pneumonia has never been reported. So, we hereby report first case of MFS which was associated with ventilator-associated pneumonia (VAP). 


\section{Case Presentation}

A 22-year-old male, with a known case of temporal lobe epilepsy since childhood and mental retardation, was on sodium valproate and Levetiracetam for his epilepsy. He presented to the emergency room complaining of three episodes of generalized tonic clonic seizure within 15 minutes without returning to baseline consciousness between the seizures. The patient was sedated, intubated and admitted to the Intensive Care Unit (ICU) as a case of status epilepticus. Two days later, he developed a fever $>38^{\circ} \mathrm{c}$ associated with increased tracheobronchial secretions. Relevant septic work-up was sent and it showed leukocyte count of $18.3 \times 10^{9} / \mathrm{mL}$ and culture of tracheobronchial secretions showed growth of Pseudomonas aeruginosa. A chest X-ray showed new infiltrates in right lower lobe consolidation (Figure 1) which could be sign of aspiration but risk of aspiration was low in this case because all measures to prevent aspiration were taken including a semirecumbent position, surveillance of enteric feeding and use of promotility drugs. Diagnosis of VAP was made based on the development of pneumonia that occurred more than 48 hours after he was intubated and mechanically ventilated. He improved clinically so he was extubated. He stayed in ICU for 5 days and then was transferred back to a ward. He

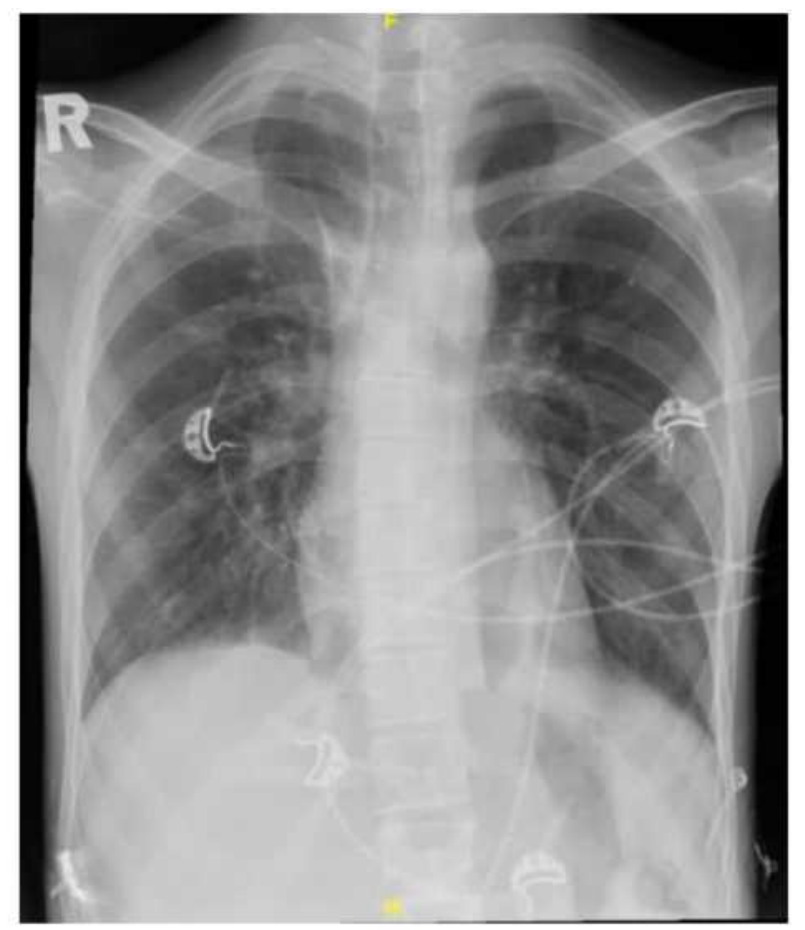

Figure I Chest X-ray showing right lung lower lobe consolidation. again became febrile and hypotensive on the second day of his stay on the ward. So, he was transferred back to ICU and antibiotics were modified to a combination of PiperacillinTazobactam and Vancomycin. He did not require mechanical ventilation this time and started to show improvement on second day of stay in ICU. He became afebrile and was transferred out of ICU to ward after four days' stay in ICU. Upon transfer to a ward his vital signs were stable, he was afebrile and maintaining oxygen saturation on room air. $\mathrm{He}$ had returned back to his baseline condition prior to admission. After 5 days on the ward he gradually developed progressive bulbar weakness and ataxia. On examination, his vital signs were a pulse of 48 beats per minute, blood pressure of $96 / 63 \mathrm{mmHg}$ and temperature of $36.9^{\circ} \mathrm{c}$. On neurologic examination he had ophthalmoplegia, finger to nose dysmetria, dysdiadochokinesia, abnormal heel to shin test, generalized areflexia and difficulty in standing and needed full support to stand up. The rest of the neurologic examination was unchanged from his baseline examination. The patient underwent a lumbar puncture and a CSF analysis showed proteins $100 \mathrm{mg} / \mathrm{dl}$ and a white cell count of 10 . MRI brain and spine was also done which shows redemonstration of atrophic left hippocampus with abnormal $\mathrm{T} 2$ and Flair hyperintense signals due to mesial temporal sclerosis (Figure 2A and B). Levels of anti-ganglioside antibodies were: Anti-GD1b 198, Anti-GD1a 175, AntiGM1 154, Anti-GM2 41 and Anti-GQ1b <30. Reference range for all these antibody is less than 30 . Since the antibody tests were strongly positive electrophysiological studies were not performed which might have been compromised due to the patient being mentally challenged and thus unable to cooperate. A diagnosis of sero-negative MFS variant of GBS was made and the patient received 5 sessions of intravenous immunoglobulin (IVIG). After 3 days his hemodynamic status improved to give a blood pressure reading of $113 / 67 \mathrm{mmHg}$, his heart rate was 68-75 beats per minutes but other neurologic symptoms did not improve. The patient was monitored closely until his hemodynamic status stabilized. Once hemodynamics were stable, the rehabilitation team was consulted. Written informed consent has been provided by the patient to have the case details and any accompanying images published, institutional approval is not required for case report publication.

\section{Discussion}

MFS, a clinical triad of ophthalmoplegia, areflexia and ataxia, is one of the regional variants of GuillainGBS 


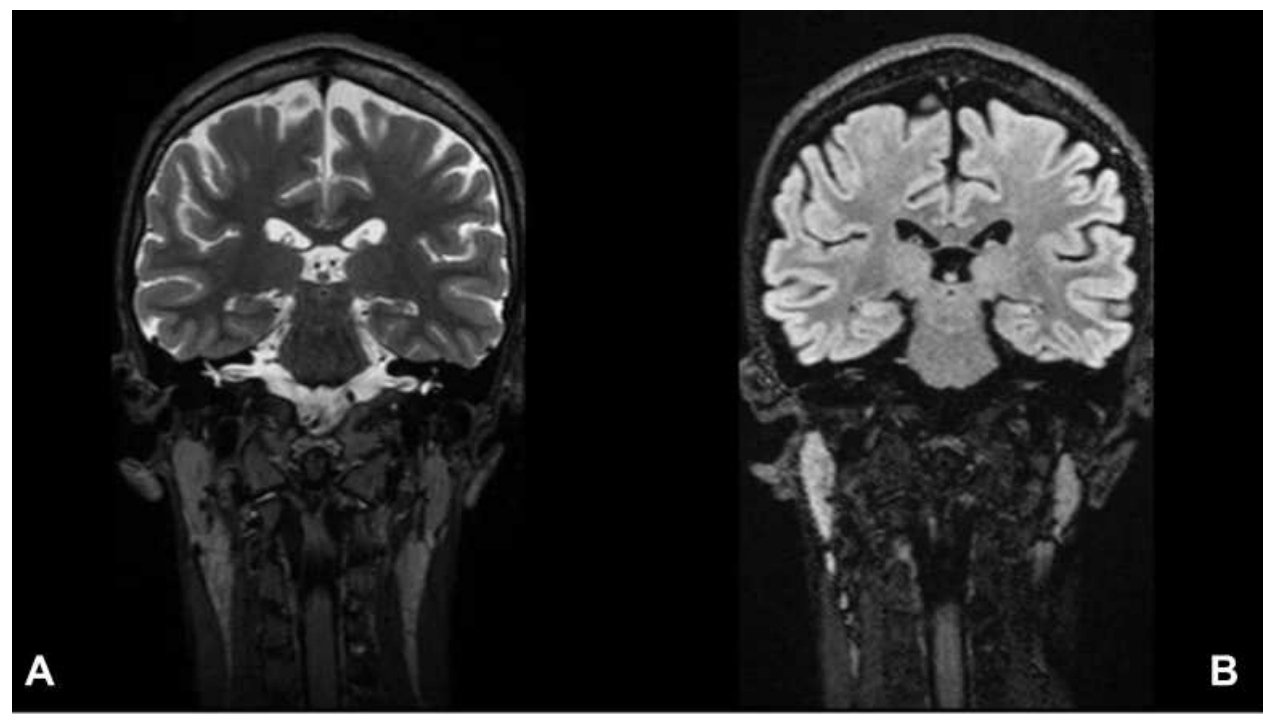

Figure 2 Mesial temporal sclerosis. Coronal brain MRI T2 sequence (A) and FLAIR sequence (B) showing left hippocampal atrophy and high signal intensity.

that might account for a -quarter of all cases of GBS, especially in Asian countries. There is history of an antecedent upper respiratory tract infection in up to two thirds of MFS cases and the most common pathogens related to MFS are Campylobacter jejuni, Haemophilus influenzae, Cytomegalovirus and Mycoplasma Pneumoniae, in that order. ${ }^{1-3}$ It is hypothesized that infection triggers an aberrant immune response producing autoantibodies via molecular mimicry. ${ }^{6,7}$

In this case, the patient presented with a classic triad of MFS associated with bulbar symptoms. Although the diagnosis of MFS is clinical, some supportive investigations help to confirm the diagnosis such as elevated proteins in CSF and the presence of anti-GQ1b antibody which is found in $85 \%$ of cases. ${ }^{8}$ However, in our case anti-GQ1b antibodies were absent but antibodies against other components of Ganglioside complex were elevated, including anti-GM1, anti-GM2, anti-GD1a and antiGD1b were found to be very high. Hence diagnosis of sero-negative MFS was made. In such cases, Koja et al found that $\operatorname{IgG}$ antibodies against other components of Ganglioside complex including GM1B, GD1a, GD1b, GD1c, GalNAc-GM1B and GT1a are found to be elevated, the majority of the clinical features were similar between GQ1b-seronegative and GQ1b-seropositive MFS. ${ }^{9}$ The nerve conduction study is usually normal in MFS but some cases might have discrete changes. ${ }^{10-12}$ Our case demonstrated autonomic dysfunction in form of bradycardia and hypotension. Although subclinical autonomic dysfunction is reported in as many as $83 \%$ of
MFS patients, overt autonomic dysfunction is rarely seen. ${ }^{13}$ However, sometimes it can be severe enough to warrant interventions like cardiac pacemaker insertion. ${ }^{14-16}$

The patient developed MFS 10 days after he was diagnosed with VAP. To our knowledge this is first case report of the MFS variant of GBS being triggered by VAP. There is one case report of an adult diabetic patient who developed GBS after bacterial meningitis secondary to chronic supportive otitis media (CSOM) with unknown causative organism. The causative organisms for CSOM are mainly Proteus mirabilis, Pseudomonas aeruginosa, Staphylococcus aureus and anaerobes. It was proposed in that case that GBS was likely triggered by one of these organisms. ${ }^{17}$

GBS following VAP is not reported in literature. Our case shows onset of MFS variant of GBS following VAP. This case suggests a new triggering factor for GBS. Physicians should have high index of suspicion for GBS in a patient who develops acute, progressive muscle weakness post VAP and administer IVIG or plasmapheresis as soon as possible. Further research is needed to investigate Pseudomonas aeruginosa as a triggering factor of GBS.

\section{Conclusion}

MFS is an immune-mediated entity which is usually triggered by upper respiratory tract infection but in rare cases it can be consequence of pneumonia, including VAP. Further research is needed to establish a link between MFS and VAP. 


\section{Funding}

There is no funding to report.

\section{Disclosure}

The authors reported no conflict of interest in relation to this work.

\section{References}

1. Doets AY, Verboon C, van den Berg B, et al. Regional variation of Guillain-Barré syndrome. Brain. 2018;141(10):2866-2877. doi:10.1093/brain/awy232

2. Mori M, Kuwabara S, Fukutake T, Yuki N, Hattori T. Clinical features and prognosis of Miller Fisher syndrome. Neurology. 2001;56 (8):1104-1106. doi:10.1212/WNL.56.8.1104

3. Ito M, Kuwabara S, Odaka M, et al. Bickerstaff 's brainstem encephalitis and Fisher syndrome form a continuous spectrum: clinical analysis of 581 cases. $J$ Neurol. 2008;255:674-682. doi:10.1007/s00415008-0775-0

4. Lee SY, Lee YH, Chun BY, et al. An adult case of fisher syndrome subsequent to mycoplasma pneumoniae infection. J Korean Med Sci. 2013;28(1):152-155. doi:10.3346/jkms.2013.28.1.152

5. Bernal Sánchez-Arjona M, Franco Macías E, Villalobos-Chaves F. [Miller Fisher syndrome in the course of an acute pneumonia by Mycoplasma pneumoniae. Rev Neurol. 2003;36(3):235-237. doi:10.33588/rn.3603.2002236

6. Jasti AK, Selmi C, Sarmiento-Monroy JC, Vega DA, Anaya JM, Gershwin ME. Guillain-Barré syndrome: causes, immunopathogenic mechanisms and treatment. Expert Rev Clin Immunol. 2016;12 (11):1175-1189. doi:10.1080/1744666X.2016.1193006

7. Nobile-Orazio E. The complement story in Guillain-Barré syndrome: from pathogenesis to therapy. Lancet Neurol. 2018;17(6):483-485. doi:10.1016/S1474-4422(18)30144-3
8. Nishimoto Y, Odaka M, Hirata K, Yuki N. Usefulness of anti-GQ1b IgG antibody testing in Fisher syndrome compared with cerebrospinal fluid. Neuroimmunol. 2004;148(1-2):200-205. doi:10.1016/j. jneuroim.2003.11.017

9. Koga M, Gilbert M, Takahashi M, et al. GQ1b-seronegative Fisher syndrome: clinical features and new serological markers. $J$ Neurol. 2012;259(7):1366-1374. doi:10.1007/s00415-011-6360-y

10. Leonhard SE, Mandarakas MR, Gondim FA, et al. Diagnosis and management of Guillain-Barré syndrome in ten steps. Nat Rev Neurol. 2019;15(11):671-683. doi:10.1038/s41582-019-0250-9

11. Van Den Berg B, Walgaard C, Drenthen J, Fokke C, Jacobs BC, Van Doorn PA. Guillain-Barré syndrome: pathogenesis, diagnosis, treatment and prognosis. Nat Rev Neurol. 2014;10(8):469. doi:10.1038/ nrneurol.2014.121

12. Khadilkar SV, Yadav RS, Patel BA. Miller Fisher Syndrome. In: Neuromuscular Disorders. Singapore: Springer; 2018:531-534).

13. Lyu RK, Tang LM, Hsu WC, Chen ST. Quantitative cardiovascular autonomic function study in Fisher syndrome. J Neurol Neurosurg Psychiatry. 2002;73(3):333-335. doi:10.1136/jnnp.73.3.333

14. Yuan CL, Wang YJ, Tsai CP. Miller fisher syndrome: a hospital-based retrospective study. Eur Neurol. 2000;44(2):79-85. doi:10.1159/ 000008201

15. Kordouni M, Jibrini M, Siddiqui MA. Long-term transvenous temporary pacing with active fixation bipolar lead in the management of severe autonomic dysfunction in Miller-Fisher syndrome: a case report. Int $J$ Cardiol. 2007;117(1):e10-e12. doi:10.1016/j. ijcard.2006.07.086

16. Kaushik P, Cohen AJ, Zuckerman SJ, et al. Miller fisher variant of guillain-barre syndrome requiring a cardiac pacemaker in a patient on tacrolimus after liver transplantation. Ann Pharmacother. 2005;39 (6):1124-1127. doi:10.1345/aph.1E676

17. Ding L, Chen Z, Sun Y, et al. Guillain-Barré syndrome following bacterial meningitis: a case report and literature review. BMC Neurol. 2018;18. doi:10.1186/s12883-018-1211-4
International Medical Case Reports Journal

\section{Publish your work in this journal}

The International Medical Case Reports Journal is an international, peer-reviewed open-access journal publishing original case reports from all medical specialties. Previously unpublished medical posters are also accepted relating to any area of clinical or preclinical science. Submissions should not normally exceed 2,000 words or 4 published pages including figures, diagrams and references. The manuscript management system is completely online and includes a very quick and fair peer-review system, which is all easy to use. Visit http://www.dovepress.com/testimonials.php to read real quotes from published authors 